\title{
(n) \\ Sleep habits, quality and chronotype of Paralympic athletes
}

\author{
Dekkel Bachar ${ }^{1,2}$, Davidah Koseff ${ }^{2}$, Osnat Fliess Douer ${ }^{2,3}$ \\ Received: $9^{\text {th }}$ September 2020; Accepted: $30^{\text {th }}$ November 2021; Published: $14^{\text {th }}$ February 2022
}

\begin{abstract}
This study aimed to understand the sleeping habits of Israeli Paralympic Athletes as a pre-intervention stage to implement sleep hygiene programs. The Pittsburgh Sleep Quality Index questionnaire was used to assess sleep quality. The Epworth Sleepiness Scale was used to determine sleepiness throughout the day. Chronotype was detected using the Horne and Östberg Questionnaire. Paraathletes who were expected to represent Israel at the Tokyo 2020 games were approached via email. The surveys were collected online between April and August, 2018. Para-athletes $(\mathrm{n}=52$, male $=32$, female $=20$, mean age $=31.2 \mathrm{y}, \mathrm{SD}=11.9$, from 13 different sport disciplines) completed the online surveys. One-way ANOVA test was used to compare sleep efficiency between the sleepy and non-sleepy groups. MANOVA test was performed to evaluate differences between sleep efficiency, daytime dysfunction and latency between the good and poor sleep quality groups. Non-parametric tests were conducted to analyze the association between the results of the three questionnaires and each sample characteristics. Under a third (31\%, $\mathrm{n}=16$ ) of the athletes slept between 6.5 to 7 hours per night, and a further $29 \%$ $(n=15)$ slept less than 6 hours. From the MANOVA analysis, athletes with poor sleep quality had statistically significantly lower sleep efficiency $(\mathrm{p}=.028, \mathrm{~F}(1,50)=5.11$, medium effect size: $\left.\eta_{p}^{2}=.093\right)$, greater daytime dysfunction $(\mathrm{p}<.001, \mathrm{~F}(1,50)=$ 14.19, large effect size: $\left.\eta_{p}^{2}=.221\right)$, and greater sleep latency $(\mathrm{p}<.001, \mathrm{~F}(1,50)=$ 15.08, large effect size: $\eta_{p}^{2}=.232$ ), than athletes with good sleep quality. Of the athletes, 33\% reported having 'moderate to excessive daytime sleepiness', 46\% did not train at times that match their chronotype. The results of this study may aid in planning effective intervention methods to improve athletes' sleep quality.
\end{abstract}

Keywords: Para-athlete; Sleepiness; Sleep questionnaires; Tokyo 2020

\section{Introduction}

Sleep has been recognized as a significant component in athlete training regime, and it is suggested to be one of the most effective recovery strategies available to athletes (O'Donnell et al., 2018). Although there is a large body of research available about sleep among "able-bodied" athletes, in the Paralympic field, this topic has not been sufficiently researched thus far. Elite athletes, both Olympians and Paralympians, are presumed to face similar issues regarding their sleeping habits, especially prior to major competitions (Juliff et al., 2015). Sleep is a reversible behavioural state where an individual is disengaged and unresponsive to his or her external environment. Sleep can drastically improve athletic performance and recovery (Halson, 2014). Athlete performance is generally defined as goaldirected behaviour that requires physical and mental effort (Halson, 2014). The study of 
Paralympic athletes can add to the existing knowledge focusing on the sleep habits of elite athletes, as there are various other factors impacting their sleep patterns. These factors include, amongst others, accessibility, transport and communication challenges, and pain caused by health conditions hindering training schedules (Rodrigues et al., 2017). On average, an individual's night sleep is divided into 90-minute cycles split by periods of nonrapid-eye-movement (NREM) states and rapid-eye-movement (REM) (Fullagar et al., 2015). NREM sleep assists with energy conservation and nervous system recuperation (Fullagar et al., 2015). NREM sleep is subdivided into 3 phases. Each phase represents the relative depth of sleep and offers particular characteristics in the brain wave and muscle tones (Brinkman et al., 2020). REM sleep is associated with higher cognitive activity. It is anatomically identified by muscle atonia, periodic brain activation, localized recuperative processes, and emotional regulation. REM (the dreaming phases) is defined by the American Academy of Sleep Medicine, as "the stage of sleep with the highest brain activity, characterized by enhanced brain metabolism and vivid hallucinatory imagery or dreaming” (American Academy of Sleep Medicine, 2001, p.349). Sleep has a direct impact on individual physiological and psychological ability and development regarding daily tasks and athletic performance (Fullagar et al., 2015). When asleep, 95\% of the daily production of growth hormones are released during the NREM sleeping stage, meaning that this is the most critical time for the body to actively repair and restore itself (O'Donnell et al., 2018). During the REM phase, memory consolidation and the learning of motor skills are developed. Due to the nature of sport and high performance, athletes require high levels of cognitive demand in addition to constant and consistent motor learning to ultimately succeed. Therefore, the REM phase is a critical time for the athletes to develop their cognitive skills, and this will significantly impact their performance (O’Donnell et al., 2018).

The term sleep efficiency is often defined as the ratio between total time asleep and the time spent in bed (Reed \& Sacco, 2016). When assessing the sleep efficiency of an athlete three primary factors are taken into consideration; sleep length (total sleep duration; hours/night, plus naps), sleep quality (i.e., the experience and perceived adequacy of sleep), and sleep phases (circadian timing of sleep) (Fullagar et al., 2015; Doherty et al., 2019). Halson (2014) describes some of the major issues that impact sleep efficiency in athletes as, unusual surroundings, noise, nervousness before competition, poor routine that could be caused by early or late training, and competition times. Poor sleeping habits can include the use of technology before bed, nocturnal waking to use the restroom, caffeine, and excessive overthinking. These issues can negatively impact both their mental and physical health, and have significant consequences on the quality and time of athletes' post-performance recovery (Fullegar et al., 2015). 'Sleep quality' is defined as the quantitative measures of sleep, including sleep duration, latency, number of arousals throughout the night, and individual opinions to whether their sleep was 'deep' or 'restful' (Buysse et al., 1988).

Silva and colleagues conducted a study among Brazilian Paralympic athletes aimed at evaluating athletes' sleep quality, chronotype, and anxiety levels before the Beijing 2008 games (Silva et al., 2012). Their results showed that $83 \%$ of the athletes who participated in the study demonstrated excessive daytime sleepiness and had poor sleep quality. Athletes who demonstrated poor sleep quality, in turn, demonstrated greater sleep latency. Athletes who experienced excessive daytime sleepiness had lower sleep efficiency in comparison with the non-sleepy athletes (Silva et al., 2012).

This study aimed to understand the current sleeping habits of Israeli Paralympic Athletes as a pre-intervention stage, prior to the implementation of an effective sleep hygiene program. More specifically, the purpose of this study was to evaluate the quality of sleep, sleepiness and chronotype of the athletes, as a pre-intervention step before applying 
SleepRate monitoring (sensor and application, Hypnocore Ltd), to help the athletes better prepare and qualify for the Tokyo 2020 games. SleepRate is an athlete-friendly, accurate, scientifically valid, digital solution for athletes to harness their sleep, enhance recovery, prevent injuries and optimize performance (Fliess Douer, 2019).

\section{Materials and Methods}

\section{Participant Recruitment}

Athletes belonging to the Israel National Paralympic squad ( $\mathrm{N}=87)$, participating in major competitions on the road to the Tokyo 2020 games, were invited to take part in this study via email communication. The announcement about the survey was made in March 2018, during a workshop with athletes and coaches. The surveys were collected online between April and August, 2018. All participants were fluent in Hebrew. Athletes included in the study had either physical or visual impairments. Athletes with visual impairments who needed assistance to complete the online survey were assigned a non-biased aide by the research team. The Israeli Paralympic squad does not currently have athletes with intellectual impairments (II). Therefore, athletes with II were not included in this study.

\section{Procedures}

A detailed email was sent by the researchers, who are members of the Israeli Paralympic Committee, informing the athletes about the aims and methods of the study. The ethical committee at Tel Aviv University approved this study. All the participants consented to be a part of the study and were aware of all of the procedures associated with it. The athletes had the freedom to decide whether or not they would like to continue with the study throughout the process. The study was conducted online through the use of Qualtrics (USA).

\section{Assessment Tools}

Following the collection of the athlete's demographic data (i.e., age, sport type, year of participation, etc.), a Hebrew version of three sleep-related questionnaires, as described below, was distributed in order to gather information on each athlete's current sleep state and habits (Shochat et al., 2007). The decision to use these questionnaires was based on the research conducted by Silva et al. (2012) with Brazilian Paralympic athletes. A comparison of sleep related variable data could be made between the outcome of the two studies, thus increasing knowledge in the Paralympic realm.

\section{Sleep Quality}

The Pittsburgh Sleep Quality Index (PSQI) was developed by Buysse and colleagues in 1998. It is a commonly used self-report instrument for assessing sleep quality. It is comprised of 19 items and seven domains of sleep including: subjective sleep quality, sleep latency, sleep duration, sleep efficiency, sleep disturbances, use of sleep medication, and daytime dysfunction. Each item is weighted on a $0-3$ interval scale. The global PSQI score is then calculated by adding the seven component scores together, providing an overall score ranging from o to 21; the lower the score, the healthier the sleep quality. Global score of $>5$ is generally used to indicate poor sleep (Hinz et al., 2016).

\section{Chronotype}

The Horne and Östberg (1976) Morningness and Eveningness Questionnaire (MEQ) was used to evaluate the athlete's chronotype. The MEQ is a self-assessment questionnaire that divides individuals into groups according to their preference for performing tasks in the morning or evening (i.e., at what time of the day is the athlete's practice and performance most effective). The questionnaire consists of 19 questions. Each question is scored on a 45-point scale, and the total score can range between 16 and 96. Chronotype scores were 
determined using the Horne and Östberg classification system where scores of 16-41 = evening type; 42-58 = intermediate type; 59-86 = morning type (Lastella et al., 2016).

\section{Sleepiness}

The Epworth Sleepiness Scale (ESS) was used to assess daytime sleepiness (Johns, 1991). The ESS describes eight different situations and requires participants to rank how likely they are to fall asleep in each given scenario on a scale ranging from o (no chance of dozing off) to 3 (high chance of dozing off). The sum of the scores for the eight questions ranges between 0 and 24. Scores between $0-9$ is considered to be normal; 10-24 indicate excessive sleepiness, and in these cases, an intervention is recommended (Lastella et al., 2016).

\section{Statistical Procedures}

Descriptive statistics were performed with Microsoft Excel 2018. The sample was divided into two groups; athletes with poor quality of sleep, and athletes with good quality of sleep. In order to evaluate differences between sleep efficiency, daytime dysfunction and latency between the good and poor sleep quality groups, MANOVA test was performed with IBM SPSS version 25. One-way ANOVA test was used to compare sleep efficiency between the sleepy and non-sleepy groups. As a measure of effect size, we used partial eta squared $\left(\eta_{p}^{2}\right)$. This measure describes the proportion of variance that a variable explains that is not explained by other variables in the analysis. Partial eta squared is overwhelmingly cited as a measure of effect size in the research literature. Widely used suggestions about what constitutes a large or small effect are: $\eta_{p}^{2}=.01$ small effect; $\eta_{p}^{2}=.06$ medium effect, and $\eta_{p}^{2}$ $>.14$ large effect (Richardson 2011). To analyse the association between the results of the three questionnaires, and between each sample characteristics (i.e., age, gender, level of competition, impairment, chronotype, and daytime sleepiness), non-parametric tests (Mann-Whitney and Kruskal-Wallis), and Chi-square tests of independence were conducted.

\section{Results}

A total of 52 Israeli Paralympic Athletes ( male $=32$, female $=20$, mean age $=31.2 \mathrm{y}, \mathrm{SD}$ $=11.9$ ) responded to the introductory email and completed the online surveys between April 2018 and August 2018, with a response rate of 60\%. Of the participants in this study, all athletes were identified as potential competitors representing Israel at the Tokyo 2020 games. The athletes who participated in the study competed in 13 different Paralympic sport disciplines. There were 19 (36.5\%) athletes in individual indoor sports (Boccia, Powerlifting, Swimming, Badminton, Table Tennis, Shooting), 21 (40.4\%) from team sports (Goalball, Wheelchair Basketball, Wheelchair Rugby) and 12 (23.1\%) from individual outdoor sports (Hand Cycling, Kayaking, Rowing, Wheelchair Tennis). Most athletes ( $\mathrm{n}=36,69.2 \%$ ) were international athletes and the remaining 16 athletes (30.8\%) were national level. Almost all athletes $(\mathrm{n}=44,84.6 \%)$ had physical impairments (Hypertonia, Ataxia, Athetosis, Impaired Muscle Power, Impaired Passive Range Of Movement, Limb Deficiency, Short Stature). The remaining eight athletes (15.4\%) had visual impairments.

\section{Sleep Quality}

Global PSQI mean score was low (mean $=5.1, \mathrm{SD}=2.7$ ). Based on the assessment score, 29 athletes were classified as having poor sleep quality. Under a third (31\%) of the athletes reported sleep duration of less than 7 hours per night. An additional 29\% reported that in the last month, they slept less than 6 hours per night. From the MANOVA test, using Pillai's trace, there was a statistically significant effect of sleep quality on sleep efficiency, daytime 
dysfunction, and sleep latency, $V=.46, \mathrm{~F}(3,48)=13.65, \mathrm{p}<.001$. From the separate univariate tests there were statistically significant effects on each of the MANOVA's outcome variables. Athletes with poor sleep quality had statistically significantly lower sleep efficiency $\left(\mathrm{p}=0.028, \mathrm{~F}(1,50)=5.11\right.$, with medium effect size: $\left.\eta_{p}^{2}=.093\right)$, greater daytime dysfunction $\left(\mathrm{p}<.001, \mathrm{~F}(1,50)=14.19\right.$, with large effect size: $\left.\eta_{p}^{2}=.221\right)$, and greater sleep latency ( $\mathrm{p}<.001$, $\mathrm{F}(1,50)=15.08$, with large effect size: $\left.\eta_{p}^{2}=.232\right)$. The outcome shows that the differences between athletes with poor sleep quality and good sleep quality are statistically significant and with a substantial effect size. Figure 1 depicts the differences between 'good' and 'poor' sleep quality groups for sleep efficiency, daytime dysfunction and latency (based on the PSQI scale score).
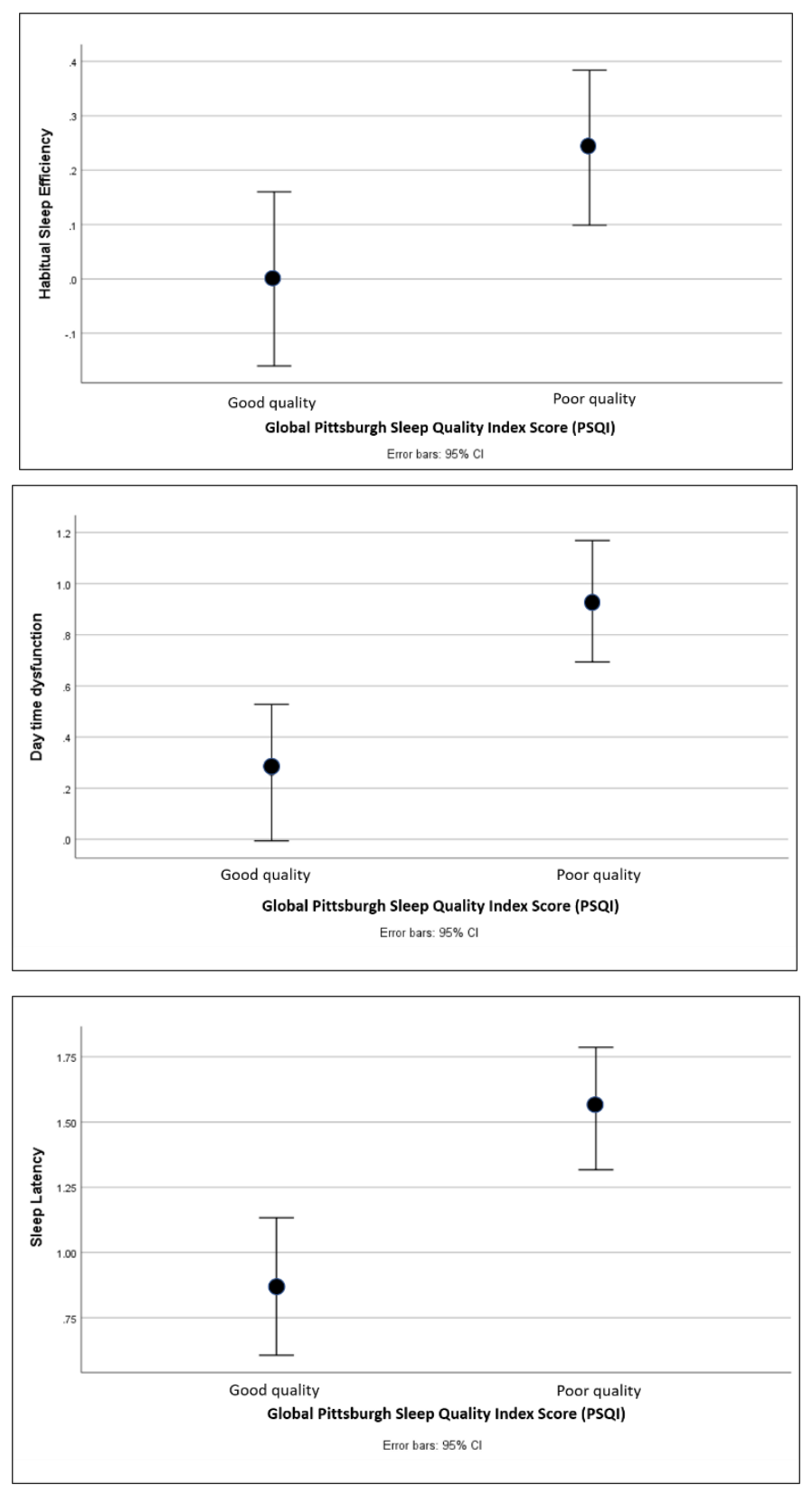

Figure 1. Differences between 'good' and 'poor' sleep quality groups based on the PSQI scale score, for sleep efficiency (top), daytime dysfunction (middle), and latency (bottom). Error bars present $95 \%$ Confidence Interval. 


\section{Sleepiness}

The results based on the ESS scale indicated that 35 of the 52 Paralympic athletes demonstrated normal daytime sleepiness. Of the athletes, $33 \%$ reported having 'moderate to excessive daytime sleepiness'. Differences in sleep quality (based on the Global PSQI score) were not statistically significant between the sleepy and non-sleepy groups ( $\mathrm{p}=.324$, $\left.\mathrm{F}=.994 . \eta_{p}^{2}=.19\right)$.

\section{Chronotype}

From the MEQ data, 60\% of the athletes were intermediate types (midday), 33\% were morning type, and $7 \%$ were evening type. After cross-referencing these results with the athlete's practice times a total of 28 of the 52 athletes (54\%) training times matched their chronotype whereas, the other $25(46 \%)$ do not train at times that matched their chronotype. Table 1 presents the PSQI, ESS, and MEQ results as well as their division into sleep quality sub-groups.

Table 1. Sleep related outcome divided into "good sleep" and "poor sleep" subgroups.

\begin{tabular}{|c|c|c|c|c|c|c|c|}
\hline \multirow[t]{2}{*}{ Assessment tool } & & \multicolumn{2}{|c|}{ Counts } & \multicolumn{2}{|c|}{$\begin{array}{c}\text { *Good sleep } \\
\text { quality }\end{array}$} & \multicolumn{2}{|c|}{$\begin{array}{l}\text { *Poor sleep } \\
\text { quality }\end{array}$} \\
\hline & & $\mathrm{N}$ & $\%$ & $\mathrm{~N}$ & $\%$ & $\mathrm{~N}$ & $\%$ \\
\hline \multirow[t]{3}{*}{ MEQ } & Morning type & 17 & 33 & 8 & 47 & 9 & 53 \\
\hline & Intermediate type & 31 & 60 & 14 & 45 & 17 & 55 \\
\hline & Evening type & 4 & 7 & 1 & 25 & 3 & 75 \\
\hline \multirow[t]{2}{*}{ ESS } & Normal daytime sleepiness & 35 & 67 & 18 & 51 & 17 & 49 \\
\hline & $\begin{array}{l}\text { Moderate/severe daytime } \\
\text { sleepiness }\end{array}$ & 17 & 33 & 5 & 29 & 12 & 71 \\
\hline \multicolumn{2}{|c|}{ PSQI global score } & 52 & 100 & 23 & 44 & 29 & 56 \\
\hline \multirow{4}{*}{ Sleep latency } & 0 & 7 & 13 & & & & \\
\hline & 1 & 26 & 50 & & & & \\
\hline & 2 & 18 & 35 & & & & \\
\hline & 3 & 1 & 2 & & & & \\
\hline \multirow[t]{4}{*}{ Sleep duration } & 0 (> 7. hours $)$ & 21 & 40 & & & & \\
\hline & 1 (6.5-7 hours) & 16 & 31 & & & & \\
\hline & 2 (5-6 hours) & 13 & 25 & & & & \\
\hline & $3(<5$ hours $)$ & 2 & 4 & & & & \\
\hline \multirow[t]{4}{*}{ Sleep efficiency } & $>85 \%$ & 46 & 88 & & & & \\
\hline & $75-84 \%$ & 5 & 10 & & & & \\
\hline & $65-74 \%$ & 1 & 2 & & & & \\
\hline & $<65 \%$ & 0 & & & & & \\
\hline Sleep & 0 & 7 & 13 & & & & \\
\hline \multirow[t]{3}{*}{ disturbances } & 1 & 40 & 77 & & & & \\
\hline & 2 & 5 & 10 & & & & \\
\hline & 3 & 0 & & & & & \\
\hline Daytime & 0 & 25 & 48 & & & & \\
\hline \multirow[t]{3}{*}{ dysfunction } & 1 & 22 & 42 & & & & \\
\hline & 2 & 4 & 8 & & & & \\
\hline & 3 & 1 & 2 & & & & \\
\hline Subjective & Very good & 8 & 15 & & & & \\
\hline \multirow{3}{*}{ sleep quality } & Fairly good & 37 & 71 & & & & \\
\hline & Fairly bad & 7 & 14 & & & & \\
\hline & Very bad & 0 & & & & & \\
\hline
\end{tabular}

PSQI - Pittsburgh Sleep Quality Index; PSQI Range: 0=no difficulty; $21=$ severe difficulties in all areas. PSQI Global Score: Good (0-4) Poor (5-21) | Sleep latency: 0= no difficulty; 3= severe difficulty. Sleep disturbances: $0=$ no difficulty; $3=$ severe difficulty $\mid$ Daytime dysfunction: $0=$ no difficulty; $3=$ severe difficulty; MEQ - Morningness - Eveningness questionnaire; ESS - Epworth Sleepiness Scale. * Based on PSQI Global Score. 
The association between the sample characteristics and sleep-related outcomes are in Table 2. The only statistically significant association that was found was between level of competition and sleep quality $\left(\chi^{2}=5.633 \mathrm{p}=.033\right)$. Two thirds $(67 \%)$ of international athletes were classified as having poor sleep quality compared with $31 \%$ of non-international athletes.

Table 2. Associations between the sample characteristics and sleep-related outcomes.

\begin{tabular}{lcccccc}
\hline & \multicolumn{2}{c}{ Chronotype } & \multicolumn{2}{c}{ Daytime sleepiness } & \multicolumn{2}{c}{ Sleep quality } \\
& Statistic & $\mathrm{p}$ & Statistic & $\mathrm{p}$ & Statistic & $\mathrm{p}$ \\
\hline Age & $\mathrm{H}=-1.58$ & $\mathrm{p}=.45$ & $\mathrm{U}=28.50$ & $\mathrm{p}=.81$ & $\mathrm{U}=411.00$ & $\mathrm{p}=.15$ \\
Gender & $\chi^{2}=1.41$ & $\mathrm{p}=.54$ & $\chi^{2}=0.78$ & $\mathrm{p}=.54$ & $\chi^{2}=0.008$ & $\mathrm{p}=.99$ \\
Level of competition & $\chi^{2}=1.59$ & $\mathrm{p}=.47$ & $\chi^{2}=0.02$ & $\mathrm{p}=.88$ & $\chi^{2}=5.63$ & $\mathrm{p}=.03$ \\
Impairment & $\chi^{2}=11.63$ & $\mathrm{p}=.48$ & $\chi^{2}=9.75$ & $\mathrm{p}=.13$ & $\chi^{2}=3.48$ & $\mathrm{p}=.74$ \\
Chronotype & - & & $\chi^{2}=.300$ & $\mathrm{p}=.86$ & $\chi^{2}=0.66$ & $\mathrm{p}=.71$ \\
Daytime sleepiness & - & & - & & $\chi^{2}=2.24$ & $\mathrm{p}=.15$ \\
\hline
\end{tabular}

H-Kruskal-Wallis; $\chi^{2}$ - chi-square; U-Mann-Whitney

\section{Discussion}

From this study, there are concerning deficits regarding the current sleep quality of the Israeli Paralympic athletes. Our study results are relatively consistent with the findings of previously published studies carried out with Paralympic athletes, such as the one by Silva and colleagues (2012).

Nearly two-thirds of the participants in this study reported that they sleep less than 6 or 7 hours per night. Sleep optimization is associated with a significant improvement in athletic performance and a reduction in the probability of injuries (O'Donnell \& Driller, 2017). Athletes with poorer sleep quality are subject to lower sleep efficiency, more daytime dysfunction and greater sleep latency (Irish et al., 2016).

According to Fullagar and colleagues, some recommend that athletes should be getting between 9-10 hours of sleep per night, while others recommend between 7-9 hours (Fullagar et al., 2015). Lack of sleep duration as reported by the athletes confirms that this phenomenon is widespread and does not relate specifically to a sport or a gender group. According to Fullagar et al. (2015), athlete performance and well-being could significantly improve if an athlete attains an adequate amount of sleep per night by extending the current amount of time he or she spends sleeping. Furthermore, sleep quality and quantity are the single best recovery methods available to elite athletes, in terms of both physiological and psychological recovery (O’Donnell \& Driller, 2017). It is well known that sleep can contribute to athletes' well-being. Other studies, such as the one conducted by Macdougall et al. (2019), suggested that additional mechanisms are needed too, such as mindfulness-acceptance interventions. Therefore, applying intervention methods that can improve the athlete's sleep latency is expected to improve his or her performance.

Although athletes reported that a vast majority of their sleep efficiency is above $85 \%$, they noted that they experience at least one sleep disturbance per night as well. These reported disturbances included distracting thoughts, noises around the house, pain, children and babies, noise from the street, pets, and the effects of menstrual cycle. These disruptions could be some of the causes of the daytime dysfunction reported by over $50 \%$ of the participants.

In our study, although the sleep quality of the athletes was poor, only $33 \%$ of the athletes reported sleepiness during the day. This is in contrast to earlier reports by Silva and colleagues (2012), where the majority of Paralympic athletes reported poor sleep quality and excessive daytime sleepiness. The ESS survey is a 'self-report' questionnaire. However, if 
quantitative objective measures had been taken to monitor sleep components, the results might have been different from those reported by the athletes.

Athletes competing in elite sports have a higher frequency, intensity, and volume of activity which have a significant impact on both their physical and mental health. Sleep interventions in athletes have proven to be successful in improving sleep quality and quantity which can lead to improved performance and recovery (Van Ryswyk, 2017). Major factors that impact the quality of one's sleep, could positively impact how one functions the following day. Ensuring that the athlete has an efficient training schedule, a healthy sleeping routine and proper nutrition could positively impact his or her level of daytime sleepiness and in turn, could possibly have a major positive impact on his or her performance.

Another aspect to consider when focusing on the improvement of quality of sleep and athletic performance, is matching practice times with athlete's chronotype. Although it is understood that chronotype is likely to have an impact on training and athletic performance, it is not yet fully understood in Paralympic athletes (Bender et al., 2018). Of the participants in this study, a third scored 'morningness' on the ESS assessment. Individuals with a morning preference are likely to maintain a regular sleep to wake schedule in comparison to those with an 'eveningness' preference (Lastella et al., 2016). Of the 52 athletes that took part in this study, 4 of them (7\%) were considered to be 'evening type' according to the ESS survey. While this number might not seem significant, three of the four athletes reported poor sleep quality. Individuals who prefer to go to sleep later (sleep time between 1:00AM9:0oAM), and still have to wake up to train early, will reduce their sleep time by an average of 3 hours per night resulting in missed REM periods, which are critical (Samuels, 2008).

As Silva and colleagues (2012) suggested, it could be effective to adjust athletes' training times to match with their chronotype. This strategy could provide better results through the effective optimization of the athlete's physical capacity and biological rhythm. Evidence suggests that troublesome sleep can occur during team travel times, jam-packed competition schedules and evening play, or training times (Fullagar et al., 2015). From a review of three studies, there were significant differences in performance, both strength and endurance, depending on the time an athlete trains in correlation with his/her circadian rhythm, related to chronotype (Rae et al., 2015). There could be a significant improvement in performance for just under half of the athletes surveyed if a change in training time would be considered.

As the athlete's career progresses, the likelihood of frequent travel for competition and training camps increases (Gupta et al., 2017). These factors can cause a major disruption in the athlete's sleep routine due to the restricted sleep times caused by scheduling and jetlag from travel (Gupta et al., 2017). It is important to note that on the road to Tokyo 2020 (which will occur in 2021 due to the COVID-19 pandemic), athletes will not be allowed to arrive more than a few days before the Paralympic Games. The short time between arrival and competition means that it will be extremely difficult for athletes to adapt to the seven-hour time zone difference naturally. It would be a major advantage if athletes arrive in Tokyo preadjusted to the new time zone to ensure that athletes have their sleep schedules properly set for the major games.

\section{Future Recommendations for Sleep Hygiene Intervention}

Following the study, each athlete received a report with his or her results and personalized recommendations in order to improve his or her quality of sleep. The athletes were assigned a meeting with a nutritionist in order to adapt their nutrition to improve their sleep.

The participants listed several factors that lead to sleep disturbances, some of which are more controllable (i.e., roommate noise, troubling thoughts) and others that are more challenging to manage (i.e., babies waking up, dogs). The most ideal environment for a good 
night's sleep includes a comfortable, cool, and dark environment with no electronic devices and minimal background noise and distractions (Watson, 2017). Watson suggests a 30 to 60-minute relaxation period prior to bedtime each night (Watson, 2017). Limiting the use of technological devices during the evening hours and limiting caffeine consumption, nicotine, cigarettes, or other stimulants can have a major impact on reducing sleep issues and disruptions. For athletes specifically, limiting the amount of training times and competitions scheduled into the night could also have a powerful effect on the individual (Watson, 2017).

Paralympic athletes have various factors in their training regimen that have the potential to create a less flexible and more time-consuming training session. This could affect their sleep quality. Some of these issues include locating accessible training facilities (and times), accessibility to transportation, or energy exertion during training (Rodrigues et al., 2017). While athletes are being told of the importance of their sleep quality and latency, the results of this study clearly indicate that sleep specific seminars, and promoting sleep hygiene interventions are needed in order to make a significant change. A previous study carried out by O'Donnell and Driller (2017), found that a 50-minute sleep-hygiene lecture (followed by a 10-minute question and answer period), focusing on the following five factors: (a) maintaining a regular bed and wake time; (b) ensuring a quiet, cool and dark environment; (c) avoidance of caffeine and other stimulants prior to sleep; (d) avoidance of light-emitting technological devices in the hours before to bedtime, and (e) the implementation of relaxation strategies before bedtime, reduced daytime sleepiness and improved the sleep quality of elite athletes.

Competitive athletes experience more pressures in relation to the demand that they put on themselves, both mentally and physically, in addition to the pressures from external environments such as media, spectators, and their teams (Gupta et al., 2017). Sleep quality can also be analyzed through impairment type. For example, from previous studies, there are greater alterations in REM sleep and some differences when dreaming, such as lack of ocular movement but olfactory, tactile and auditory adjustments when focusing on athletes with visual impairments (Silva et al., 2012). One Israeli athlete who is a veteran with partial paralysis demonstrated significant abnormal sleep behaviour. Although the reported time sleeping was upwards of 10 hours per night, his ESS score emphasized that he still experiences excessive sleepiness during the day. Self-report questionnaires are not as scientifically strong when compared to quantitative studies. However, the findings of this athlete's unique case, support the value of using self-report questionnaires regularly. This method is simple yet can reveal important information about the athlete. As for the case mentioned above, a future interventional study will focus on improving this particular elite athlete's sleep, tailoring recommendations to his exceptional condition. This study identified athletes with significant sleep disturbances. Athletes who presented with the most disturbed and poorest sleep quality in the survey, and who have the highest potential to be included in the delegation to Tokyo 2020 games were selected for the intervention with SleepRate and they have been monitored since March 2019.

\section{Limitations}

Despite the strengths of the current study in adding information on a less investigated topic, there are limitations that should be noted. The geographical scope of this research is restricted to only one country, which may lead to limited generalization of the outcomes. Therefore, conducting a comparative study combining athletes from different countries is recommended. The sample size was rather small as this study aimed at a specific selfselected group of participants. In addition, having the respondents send the questionnaire by mail, as was applied in this study, resulted in a low response rate. Finally, the information 
obtained based on participants' reports is not as reliable as the empirical measurement of objective sleep data.

\section{Conclusions}

This study was a steppingstone to understanding the sleep state of the Israeli Paralympic athletes, in order to develop tailored intervention methods to help improve each athlete's sleep quality and performance. Based on the results of this study, and through the use of various technological aids, the Israeli Paralympic research team assesses the outcomes of the interventions they intend to apply on the athletes' overall sleep quality.

\section{Perspectives}

This study contributes to the advancement of the athletic capability and capacity of the Paralympic athlete. The article is a guiding factor for intervention methods that are being used leading up to and during the Tokyo Paralympic Games, that took place in 2021. Now that there is a baseline understanding regarding the sleep condition, state, and habits of the Israeli Paralympic Athletes, carefully selected and individualized sleeping interventions are being implemented and analysed to determine if their sleep quality improves postintervention.

\section{Author affiliations:}

1 Bar Ilan University, Department of Science, Technology and Society, Ramat Gan, Israel, dekkelb@gmail.com

2 Israel Paralympic Committee, Tel Aviv, Israel, dkoseff@inter.net.il; osnat@isad.org.il

3 KU Leuven, Department of Rehabilitation Sciences, Leuven, Belgium

* Correspondence: osnat@isad.org.il; Tel.: +972 507390093

Author Contributions: Conceptualization OFD.; Methodology OFD.; Formal Analysis, OFD.; WritingOriginal Draft Preparation, DB; Writing-Review \& Editing, OFD, DK.

Funding: This research received no external funding.

Data Availability: No information has been provided.

Acknowledgments: The authors would like to thank the athletes who participated in this study and the Israeli Paralympic Committee for the support and collaboration.

Conflicts of Interest: The authors declare no conflict of interest.

\section{References}

American Academy of Sleep Medicine. (2001). International classification of sleep disorders, revised: Diagnostic and coding manual. American Academy of Sleep Medicine.

Bender, A. M., Van Dongen, H., \& Samuels, C. H. (2018). Sleep quality and chronotype differences between elite athletes and non-athlete controls. Clocks \& Sleep, 1(1), 3-12. https://doi.org/10.3390/clockssleep1010002

Brinkman, J.E., Reddy, V., \& Sharma, S. (2020) Physiology of Sleep. StatPearls Publishing. https://www.ncbi.nlm.nih.gov/books/NBK482512/

Buysse, D. J., Reynolds, C. F., Monk, T. H., Berman, S. R., \& Kupfer, D. J. (1988). The Pittsburgh sleep quality index: a new instrument for psychiatric practice and research. Psychiatry Research, 28(2)193-213. https://doi.org/10.1016/0165-1781(89)90047-4

Doherty R., Madigan S., Warrington G., \& Ellis J. (2019). Sleep and nutrition interactions: Implications for athletes. Nutrients, 11(4) 822. https://doi.org/10.3390/nu11040822

Fliess Douer ,O. (2019). Sleep habits, quality and chronotype of Paralympic athletes in the preparation for Tokyo 2020 Games. The 1oth VISTA Conference, 4-7 September, Amsterdam, The Netherlands.

Fullagar, H.H., Duffield, R., Skorski, S., Coutts, A.J., Julian, R., \& Meyer, T. (2015). Sleep and recovery in team sport: Current sleep-related issues facing professional team-sport athletes. International Journal of Sports Physiology Performance, 10(8), 950-7. https://doi.org/10.1123/ijspp.2014-0565 
Gupta, L., Morgan, K., \& Gilchrist, S. (2017). Does elite sport degrade sleep quality? A systematic review. Sports Medicine, 47(7), 1317-1333. https://doi.org/10.1007/s40279-016-0650-6

Halson, S. L. (2014). Sleep in elite athletes and nutritional interventions to enhance sleep. Sports Medicine , 44 (Suppl 1), S13-S23. https://doi.org/10.1007/s40279-014-0147-0

Hinz, A., Glaesmer, H., Brähler, E., Löffler, M., Engel, C., Enzenbach, C., Hegerl, U., \& Sander, C. (2017). Sleep quality in the general population: psychometric properties of the Pittsburgh Sleep Quality Index, derived from a German community sample of 9284 people. Sleep Medicine, 30, 57-63. https://doi.org/10.1016/j.sleep.2016.03.008

Horne, J.A., \& Ostberg, O. (1976). A self assessment questionnaire to determine morningness eveningness in human circadian rhythms. International Journal of Chronobiology, 4. 97-110.

Irish, L. A., Kline, C. E., Gunn, H. E., Buysse, D. J., \& Hall, M. H. (2015). The role of sleep hygiene in promoting public health: A review of empirical evidence. Sleep Medicine Reviews, 22, 23-36. https://doi.org/10.1016/j.smrv.2014.10.001

Johns, M. W. (1991). A new method for measuring daytime sleepiness: the Epworth sleepiness scale. Sleep, 14(6), 540-545. https://doi.org/10.1093/sleep/14.6.540

Juliff, L. E., Halson, S. L., \& Peiffer, J. J. (2015). Understanding sleep disturbance in athletes prior to important competitions. Journal of Science and Medicine in Sport, 18(1), 13-18. https://doi.org/10.1016/j.jsams.2014.02.007

Lastella, M., Roach, G. D., Halson, S. L., \& Sargent, C. (2016). The chronotype of elite athletes. Journal of Human Kinetics, 54(1), 219-225. https://doi.org/10.1515/hukin2016-0049

Macdougall, H., O’Halloran, P., Sherry, E., \& Shields, N. (2019). A pilot randomised controlled trial to enhance well-being and performance of athletes in para sports. European Journal of Adapted Physical Activity, 12(2), 7. https://doi.org/10.5507/euj.2019.006

O'Donnell, S., \& Beaven, C. M., \& Driller, M. W. (2018). From pillow to podium: a review on understanding sleep for elite athletes. Nature and Science of Sleep, 10, 243-253. https://doi.org/10.2147/NSS.S158598

O’Donnell, S., \& Driller, M.W. (2017). Sleep-hygiene education improves sleep indices in elite female athletes. International Journal of Exercise Science, 10(4). 522-530.

Rae, D. E., Stephenson, K. J., \& Roden, L. C. (2015). Factors to consider when assessing diurnal variation in sports performance: the influence of chronotype and habitual training time-of-day. European Journal of Applied Physiology, 115(6), 1339-1349. https://doi.org/10.1371/journal.pone.0219836

Reed, D. L., \& Sacco, W. P. (2016). Measuring sleep efficiency: what should the denominator be?. Journal of Clinical Sleep Medicine, 12(2), 263-266. https://doi.org/10.5664/jesm.5498

Richardson, J.T. (2011). Eta Squared and Partial Eta Squared as Measures of Effect Size in Educational Research. Educational Research Review, 6, 135-147. https://doi.org/10.1016/j.edurev.2010.12.001

Rodrigues, D., Silva, A., Rosa, J.P., Ruiz, F., Veríssimo, A., Winckler, C., Rocha, E., Parsons, A., Tufik, S., \& Mello, M. (2017). Profiles of mood states, depression, sleep quality, sleepiness, and anxiety of the Paralympic athletics team: A longitudinal study. Apunts Medicine de l'Esport 52(195), 93-101. https://doi.org/10.1016/j.apunts.2016.11.002

Samuels, C. (2008). Sleep, recovery, and performance: the new frontier in highperformance athletics. Neurologic Clinics, 26(1), 169-180. https://doi.org/10.1016/j.ncl.2007.11.012

Sateia M. J. (2014). International classification of sleep disorders-third edition: highlights and modifications. Chest, 146(5), 1387-1394. https://doi.org/10.1378/chest.14-0970

Shochat, T., Tzischinsky, O., Oksenberg, A., \& Peled, R. (2007). Validation of the Pittsburgh Sleep Quality Index Hebrew translation (PSQI-H) in a sleep clinic sample. Israel Medical Association Journal, 9(12), 853-856.

Silva, A., Queiroz, S.S., Winckler, C., Vital, R., Sousa, R.A., Fagundes, V., Tufik, S. \& de Mello, M.T. (2012). Sleep quality evaluation, chronotype, sleepiness and anxiety of 
Paralympic Brazilian athletes: Beijing 2008 Paralympic Games. British Journal of Sports Medicine, 46(2), 150-154. https://doi.org/10.1136/bjsm.2010.077016

Van Ryswyk, E., Weeks, R., Bandick, L., O'Keefe, M., Vakulin, A., Catcheside, P., Barger, L., Potter, A., Poulos, N., Wallace, J., \& Antic, N.A. (2017). A novel sleep optimisation programme to improve athletes' well-being and performance. European Journal of Sport Science, 17(2), 144-151. https://doi.org/10.1080/17461391.2016.1221470.

Watson, A.M. (2017). Sleep and athletic performance. Current Sports Medicine Reports, 16(6), 413-418. https://doi.org/10.1249/jsr.0000000000000418

(C) 2022 by the authors. Submitted for possible open access publication under the terms and conditions of the Creative Commons Attribution (CC BY) license http://creativecommons.org/licenses/by/4.o/). 